\title{
A Study of Hindrance-Caused Unscheduled Waiting Time in Railway Systems
}

\author{
Nan Cao ${ }^{1,2,3, *}$, Tao Tang ${ }^{1}$ and Chunhai Gao ${ }^{2,3}$ \\ 1 School of Electronic and Information Engineering, Beijing Jiaotong University, Beijing 100044, China; \\ ttang@bjtu.edu.cn \\ 2 National Engineering Laboratory for Urban Rail Transit Communication and Operation Control, \\ Beijing 100044, China; chunhai.gao@bj-tct.com \\ 3 Traffic Control Technology Co. Ltd., Beijing 100070, China \\ * Correspondence: nan.cao@bj-tct.com; Tel.: +86-183-2298-7160
}

Received: 5 June 2020; Accepted: 15 July 2020; Published: 17 July 2020

\begin{abstract}
In real operation, railway traffic always deviates from schedule due to exogenous disturbances and disruptions, and these deviations may cause a domino effect over the whole network. Therefore, evaluating and predicting the influence of these disturbances is of significance in train operation and dispatching. Delay is a commonly used performance indicator to describe degree of these deviations, and it may propagate to other trains. The main cause of delay is the overtime occupation on exclusive blocks. However, hindrance, which evaluates the performance of railway operation from the perspective of infrastructure occupancy, is seldom studied. In this paper, a systematical description and calculation of hindrance in railway systems is introduced from the perspective of infrastructure occupancy based on blocking time theory. The railway network was modeled as several exclusive components with running directions. Based on the precedence order and length of occupancy on conflicting infrastructure components, a sequential hindrance propagation process was identified. The proposed methods were demonstrated through the case of a reference network based on railway simulations. A relationship between the overall influence of hindrance and the length of hindrance was investigated for each infrastructure component, using statistical techniques. The results proved a clear positive relationship between the overall influence of a hindrance and its length. In addition, this relationship is affected by the location of infrastructure and amount of traffic flow in the network.
\end{abstract}

Keywords: operation quality; delay; hindrance; hindrance propagation; railway systems

\section{Introduction}

Railway is an important and irreplaceable mode of transportation due to its peculiar characteristic: high energy efficiency in handling large masses, particularly at middle and far distances, high operation speed, comprehensive safety system, and less action to climate. Service quality is an important issue to be considered in railway operation. In operational preparation phase, timetable designers try to make an efficient and stable timetable to fulfill the traffic demand as far as possible. However, unforeseen event may occur during actual operation, such as failures of rolling stocks, extended dwell times due to larger flow of passengers, speed restrictions due to infrastructure breakdowns or adverse weather, and so on. These events may lead to operation deviations from scheduled plan and then conflicts between trains. Recently, dispatching is mainly conducted through operators' judgments based on a wealth of dispatching experience in dealing with these disturbances. Therefore, it is full of meaning to develop a quantitative evaluation method to quantify the influence of disturbances and corresponding solutions to support dispatching and improve the service quality. 
Many factors may lead to deviations, which can be categorized into operational causes, passenger traffic causes, infrastructure causes, and others, such as weather condition or accidents [1,2]. It is important for operators to analyze the effects of these deviations in regard to the performance of railway operation [3]. Delay is an important performance indicator of railway operation in the set of key performance indicators [4,5]. In last decades, a lot of research has been done to comprehensively and systematically evaluate the operation quality considering delay. The study of Goverde [6] assessed delay and other performance indicators in a deterministic setting corresponding to the design times used in timetable construction. An arrival punctuality examination method was developed by Liu [7] to check on-time arrival performance based on real operation data. In the research of Peng [8], a complete evaluation index system for high-speed train diagrams was established, in which delay is used to describe passenger service quality.

With respect to the influence of deviations, passengers may miss their connections due to delay. Even worse, this influence may be propagated to other trains and spread in the network, causing negative influence [9]. Therefore, some efforts have been done to reduce the influence of these deviations. In the scheduling phase, the planning of time supplements is crucial to the robustness of timetables [10], in which recovery time is assigned for trains to recover from minor deviations, and buffer time can avoid a delay transfer to other trains [11,12]. Goverde evaluated the stability of timetable by considering the delay propagation of initial delay and explored the effect of buffer times to compensate for arrival delays [13]. A balance of recovery time and buffer time is optimized to generate a robust timetable regarding delays in railway operation [14]. In real operation, dispatchers attach importance to the influence of delays to the whole network through delay propagation. In the work of Reference [15], the railway system is modeled as a linear system in max-plus algebra, including zero-order dynamics, corresponding to delay propagation within a timetable period. Martin proposed a linear model to evaluate the influence of delay occurs at the nodes for dispatching to minimize the influence [16]. Stochastic models for delay propagation also have been studied intensively $[17,18]$. They propose to use approximations of delay distributions to reduce the computational efforts and study the error propagation for such approximations. Stochastic variations of train running time, like driver behavior, are also taken into account in the model if delay propagation occurs during the approach or departure of trains at stations [19].

In current research, a lot of work has been done related to delay, including quantitative evaluation of delay and evaluating the influence of delay. However, few analyses have considered the deviations from the perspective of hindrance in the field of dispatching support so far. Tracing the source, one of the main sources of delay in a railway system is the exclusivity of railway blocks [20]. Trains must follow others' paths sequentially, in accordance with the master timetable, until the preceding block is released. In other words, a block is not allowed to be occupied by more than one train at the same time. Once a train deviates from its scheduled timetable, it will occupy the infrastructure for longer time and hinder subsequent trains which are scheduled through the same railway infrastructure element, or it may encounter conflicts with other trains, due to railway infrastructure exclusivity. Therefore, hindrance is defined as the waiting time that arises when an infrastructure component is requested by more than one train at the same time, according to DB Richtlinie 405, which is proposed by DB Netz AG regarding to track capacity (Fahrwegkapazität). It is a microscope description of operation deviations with the scheduled plan based on microscope modeling of infrastructure.

In general, hindrances do not necessarily lead to final delay, while delay is the result of hindrances on individual block section. In other words, a train reaches its destination without delay, while it may suffer hindrances during the whole trip. Moreover, these hindrances may propagate to other trains afterward and negatively influence the whole railway network. Due to continuous execution of timetable, the hindrance may propagate to other trains, create consecutive hindrance in railway network, and finally lead to delay if headways between trains are not long enough, which greatly reduces the service quality. Therefore, it is important to study the behavior of the hindrance propagation among several trains in different operation condition. Right now, only Bendfeldt and Warninghoff 
have done some research studies about hindrance [21]. Infrastructure-related hindrance is identified and quantified based on operation records in this literature, and it is used as an indicator to evaluate the utilization of infrastructure of a heavily loaded station.

Therefore, the purpose of this paper is to develop a methodological procedure to quantify the hindrance that happens to certain infrastructure components and further study the influence of hindrance over the entire network. The proposed procedure is also intended to provide quantitative theoretical basis for dispatching. Section 2 starts with some definitions of hindrance, in which different scenarios are discussed. The calculation of hindrance is also presented in this section, which is the basic for the analysis of hindrance propagation. The hindrance propagation model and underlying theory of the algorithm are considered in Section 3. The model is established in the basis of the tree structure to describe the propagation process of hindrance during the later route. Moreover, it enables evaluating negative influence of a hindrance on certain component through backward propagation. A case study has been done on a reference network in Section 4. Finally, the main conclusions are drawn, and future researches are recommended, in Section 5.

\section{Description of Hindrance}

\subsection{Definition of Hindrance}

As mentioned above, the hindrance is the source of delay, and it leads to negative impacts due to the complex coupling between trains. According to the guideline 405 of Deutsche Bahn (DB) for Infrastructure Capacity Analysis, hindrance is the waiting time arisen when an infrastructure component is requested by more than one train at the same time. In this paper, Infrastructure component is the minimum exclusive infrastructure element with direction that hindrance occurrence.

The requests are beyond the service capacity of the infrastructure component, so that at least one request cannot be satisfied immediately. Infrastructure component is the minimum exclusive infrastructure element with direction that hindrance occurrence. Hindrance is the original and initial result of route conflict which causes waiting time of trains on infrastructure component and may finally result in railway delays in the complex railway operation situation.

Figure 1 gives an example to explain the definition of hindrance. In this example, train 2 applies the utilization of infrastructure component according to the scheduled timetable and real operation condition. However, the request cannot be fulfilled, since the infrastructure component is occupied by train 1 at this time point, and train 2 has to wait the assignment until train 1 releases the required infrastructure component. In this paper, the train which occupies the request infrastructure component is defined as the hindering train, like the train 1 in Figure 1; and the train forced to wait is called the hindered train, as train 2 in Figure 1.

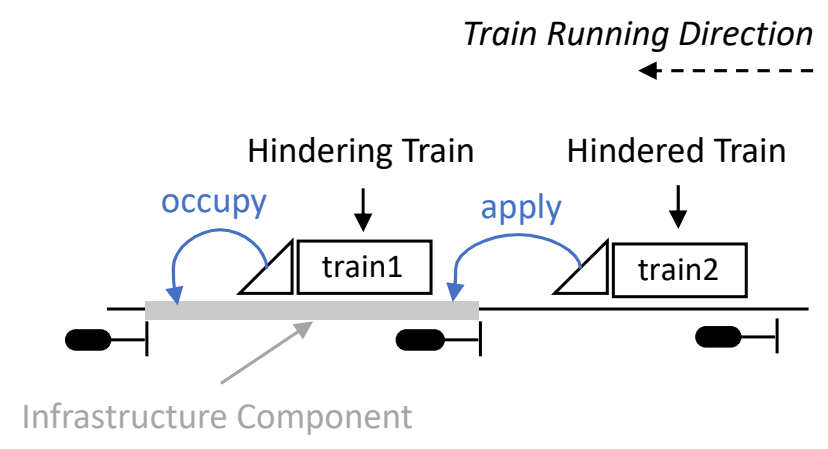

Figure 1. An example of hindrance.

Hindrance is the competition of signals in reality. According to the conflict theory of Martin [15], all conflicts occur within five categories based on running direction: following, filter out, merge, opposite without priority decision, and opposite with priority decision. Similarly, hindrance also 
occurs in the above five scenarios. In this paper, following and merge scenarios are analysis for hindrance arisen and hindrance propagation as examples, since they are the two most common and effective scenarios. For the rise and propagation of hindrance in other scenarios, the same analytical method can be employed.

\subsubsection{Following}

For "following" hindrance scenario, the sequence of two trains is determined in advance, and temporary change during operating is impossible because of the components' layout. The hindered train has to wait until the hindering train releases the infrastructure component, inducing extra waiting time of the hindered train before the requested infrastructure component. The left panel in Figure 2 is an example of "following" hindrance scenario.

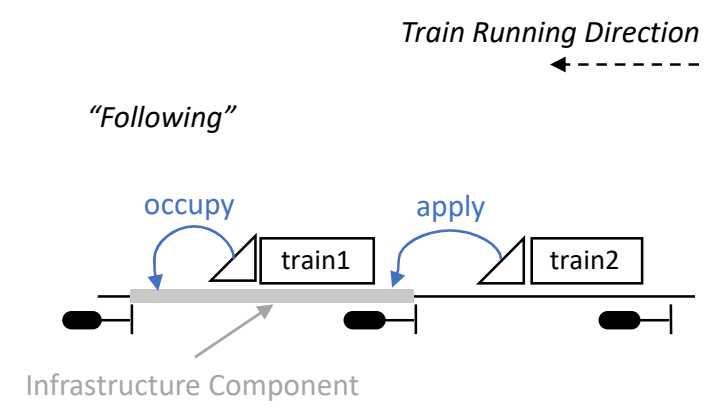

(a)

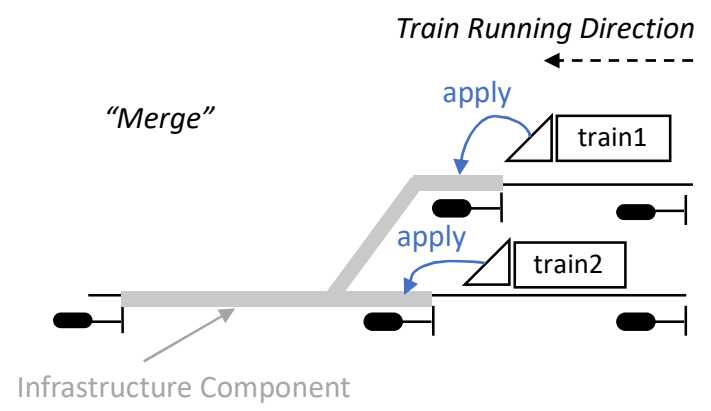

(b)

Figure 2. An example of conflict scenario: (a) "following" and (b) "merge".

\subsubsection{Merge}

As shown in Figure 2, in "merge" hindrance scenario, the trains are in a relationship of competition, and the sequence of them depends on the dispatching decision. In this case, dispatching decisions should be made according to certain dispatching rules, like minimize the negative influence to the entire railway network. If train 1 gets the assignment of infrastructure component, train 2 would be hindered by train 1 , and vice versa (i.e., train 1 would be hindered by train 2 ).

During the whole train route, a train may be hindered by different trains on different components. Moreover, more than two trains compete may occur in the hindrance conflict scenarios Merge. Consequently, some hindered train will be hindered by more than two trains in a complex situation. In order to show out the source of certain hindrance explicitly, individual hindrance is proposed in this paper to describe the direct relationship between two conflicting trains. Individual hindrance is defined as the waiting time of a train hindered by a certain train on a special infrastructure component. For each individual hindrance, it has the features: the hindered train, the infrastructure component on which the hindered train occupies the hindering train, the infrastructure component on which the hindering train occupies, and the length of waiting time of hindered train caused by hindering train. As shown in Figure 3, train 2 is hindered by train 1 on infrastructure component 2 from t 1 to 3 . Meanwhile, train 3 is hindered by two trains: first it is hindered due to the occupation of train 1 on infrastructure component 1 from $t 2$ to $t 3$; and then it is hindered by train 2 from $t 3$ to $t 5$.

\subsection{Calculation of Hindrance}

Actually, the hindrance is a kind of block source competition, which occurs in the case there is an overlapping between occupying times of components sharing same end point. According to the definition of individual hindrance, the length of hindrance is the difference of actual occupancy time and scheduled occupancy time on a certain infrastructure element. The hindrance can be divided into several individual hindrances based on the difference in hindering train. 


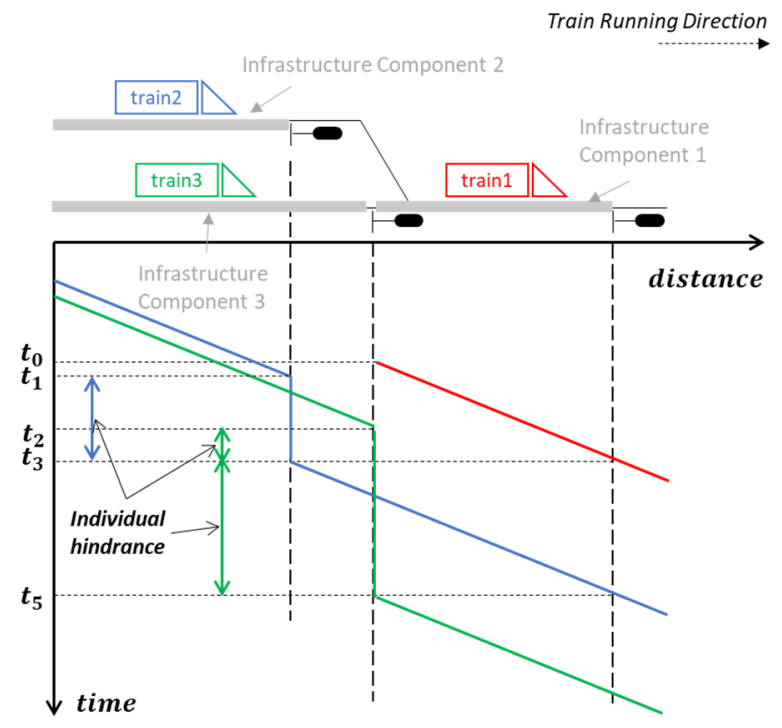

Figure 3. An example of individual hindrance.

\subsubsection{Symbols}

For the calculation of individual hindrance, the scheduled timetable and real operational timetable are required, in which the time points of all trains through each signal should be recorded. The following symbols are used for the calculation of individual hindrance:

$i$ : the serial number of train (with train class);

$s s_{j}$ : $\quad$ the start-signal ID of infrastructure component $j$;

es $j$ : the end-signal ID of infrastructure component $j$;

$s t_{s, i, j}: \quad$ the scheduled begin time point for train $i$ to run through infrastructure component $j$;

$s t_{e, i, j}: \quad$ the scheduled end time point for train $i$ to run through infrastructure component $j$;

$r t_{s, i, j}:$ the real begin time point for train $i$ to run through infrastructure component $j$;

$r t_{e, i, j}:$ the real end time point for train $i$ to run through infrastructure component $j$;

$H_{i, j}: \quad$ the length of hindrance for train $i$ on infrastructure component $j$;

$r a_{i, j}$ : the real time point for train $i$ to apply infrastructure component $j$;

$r p_{i, j}: \quad$ the real time point for train $i$ to get the permit of infrastructure component $j$;

$R O_{i, j}$ : the real occupation time for train $i$ on infrastructure component $j$;

$S O_{i, j}:$ the scheduled occupation time for train $i$ on infrastructure component $j$;

$s i h_{i, k}$ : the start time point of individual hindrance between train $i$ and train $k$;

$e i h_{i, k}$ : the end time point of individual hindrance between train $i$ and train $k$;

$I H_{i, k, j}:$ the individual hindrance of train $k$ on infrastructure component $j$ caused by train $i$;

$\mathrm{OH}_{i, j}$ : the overall influence of hindrance of train $i$ on infrastructure component $j$;

$O P R_{i, j}$ : the overall propagation rate of hindrance $H_{i, j}$.

In actual operation, this situation exists that a train applies for multiple consecutive components at same time point. Meanwhile, the release times are different for these infrastructure components, which means the occupancy of the infrastructure component is not absolutely independent of other components. In other words, these infrastructure components are treated as a whole and assigned the component number of the final infrastructure component. Correspondingly, for the parameters related to these components, e.g., start and end point, the time points through these points should be updated accordingly.

Assumption 1: The calculation of hindrance is based on simulation results or actual operation data, in which the sequences of trains through a certain component are decided and known in advance. 
Assumption 2: In actual operation, a train has to prepare to slow down and stop at the next signal when it meets a yellow signal, which happens if there is another conflicting train ahead occupying the requested infrastructure component. Hereby, we assume that the speed profile in an infrastructure component is consistent with that in the scheduled timetable, which does not inflect the influence of delay, deceleration, and acceleration on the running time.

Assumption 3: The overlap and release principle of infrastructure component are different for different hindrance scenarios. In this paper, the overlaps for different hindrance scenario are not considered in blocking time calculation.

\subsubsection{Method}

Figure 4 gives an example of the detail occupation situation in "following" and "merge" hindrance scenarios. As shown in Figure 4, the application time points and the application permit time points for certain infrastructure component determines length of the hindrance. Afterward, further comparing the occupation situation of requested infrastructure components during this time period should be done to definitely settle the individual hindrances. If the requested infrastructure component is occupied by a certain train during this time period, this train could be set as the hindering train on this infrastructure component.
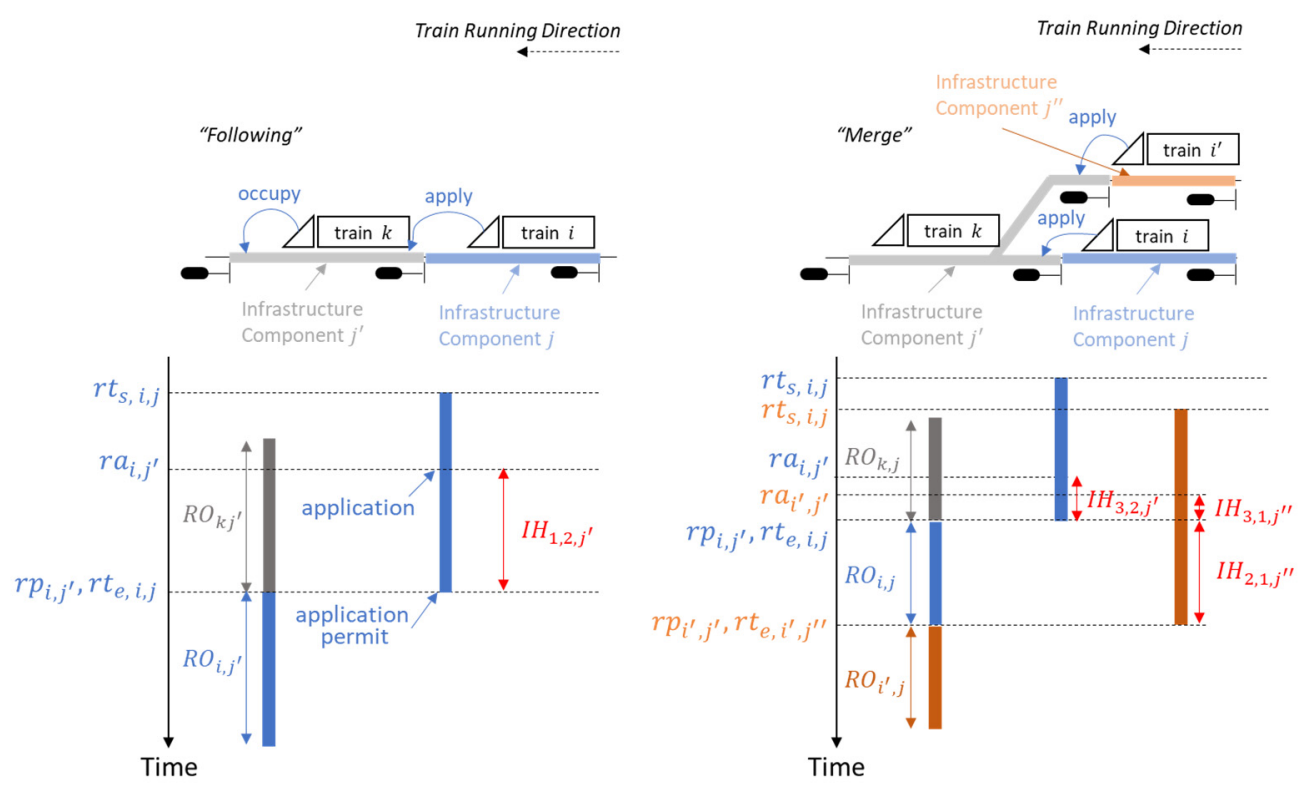

Figure 4. The occupancy of infrastructure component in "following" and "merge" hindrance scenarios.

Therefore, the total hindrance time for target train should be determined first. Then, through comparing the hindrance with the occupancy situation of the requested infrastructure component, the hindrance could be assigned to different trains for "following" and "merge" hindrance scenarios.

Figure 5 shows six scenarios of individual hindrance occurrence between two conflicting trains. Individual hindrance is marked by two time points: "Begin" and "End". The time point "Begin" is the maximum value (latest occurring time) of the start of the hindering train occupancy on hindering occupancy element and the application time of hindered train for next component. The time point "End" is the minimum value (earliest occurring time) of the release time for the hindered train to next component and the beginning time point of the next consecutive occupancy on potential hindering component. 


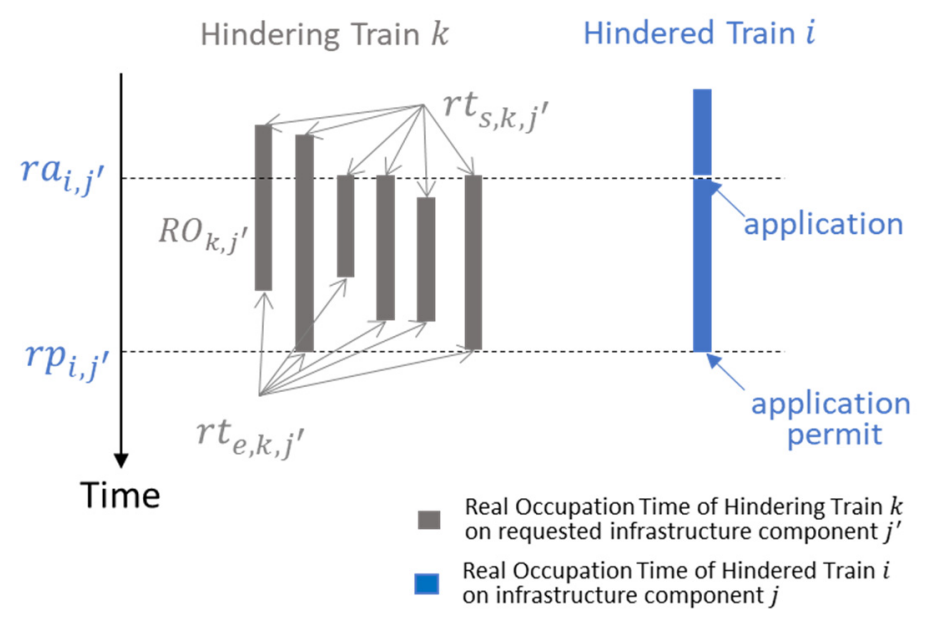

Figure 5. Six scenarios of individual hindrance arisen between two conflicting trains.

The process to calculate the length of individual hindrance is conducted with the following steps: 1. Check whether a train is hindered.

First of all, whether train $i$ is hindered should be checked by comparing operational timetable with scheduled timetable on certain infrastructure component. The length of hindrance on infrastructure component $j$ can be calculated by using the following Equation (1):

$$
H_{i, j}=R O_{i, j}-S O_{i, j}=\left(r t_{e, i, j}-r t_{s, i, j}\right)-\left(s t_{e, i, j}-s t_{s, i, j}\right)
$$

If $H_{i, j} \leq 0$, it means there is no hindrance for train $i$ on component $j$. In contrast, $H_{i, j}>0$ means that train $i$ occupies the infrastructure component $j$ longer than scheduled. In other words, train $i$ is hindered on component $j$. Further calculation to determine the individual hindrances for train $i$ should be done in this case.

2. Determine the real application time point for certain infrastructure component (refer to Figure 4).

This step is to figure out the hindered time period of the hindered train. According to Assumption 2 , the hindered train needs the scheduled occupation time to reach the next infrastructure component and apply the assignment to run through it. Thus, the real application time point of train $i$ for next infrastructure component $j^{\prime}$ is determined by Equation (2):

$$
r a_{i, j^{\prime}}=r t_{s, i, j}+\left(s t_{e, i, j}-s t_{s, i, j}\right)
$$

The real time point of permitting the application is actually the end of real occupation:

$$
r p_{i, j^{\prime}}=r t_{e, i, j}
$$

3. Determine the "Begin" and "End" time points for each individual hindrance (refer to Figure 5).

As discussed above in Figure 5, if an overlap could be found between the occupation of train $k$ on requested infrastructure component $j^{\prime}$ and the hindered time period of train $i$ on infrastructure component $j$, it suppose that an individual hindrance of train $i$ caused by train $k$ on infrastructure component $j^{\prime}$, which is transformed to the inequality below:

$$
r a_{i, j^{\prime}}<r t_{e, k, j^{\prime}} \text { AND } r p_{i, j^{\prime}} \geq r t_{e, k, j^{\prime}}
$$

The start and end time points of individual hindrance between train $i$ and train $k$ can be calculated by Equations (5) and (6):

$$
\begin{aligned}
& \operatorname{sih}_{i, k}=\max \left(r a_{i, j^{\prime}}, r t_{s, k, j^{\prime}}\right) \\
& e i h_{i, k}=\min \left(r p_{i, j^{\prime}}, r t_{e, k, j^{\prime}}\right)
\end{aligned}
$$


4. Calculate the length of individual hindrance.

The length of individual hindrance between train $i$ and train $k$ is the difference between the start and end time point of individual hindrance:

$$
I H_{i, k}=s i h_{i, k}-e i h_{i, k}
$$

\section{Propagation of Hindrance}

\subsection{Description}

Hindrance propagation is the result of a continuous train running on exclusive tracks. When conflict happens among trains, especially in "merge" hindrance scenario, the future influence of a hindrance to the entire railway network is an important reference for the dispatcher to make the decision. In order to explore the future influence of hindrance, hindrance propagation behavior should be studied first.

In order to better explain the process of hindrance propagation, the hindrance that first occurs in the network is called the initial hindrance, and the secondary hindrance is the hindrance caused by other trains due to insufficient headway. The propagation of hindrance carries out in two directions: first, the initial hindrance may lead to successive hindrances of subsequent trains backward, starting from the initial hindered component, which is called the backward propagation in this paper; secondly, some new hindrances may occur relating to the hindered train during later route move forward. However, this forward hindrance only makes negative effects on the hindered train itself, and this hindrance could be eliminated through recovery time. In contrast, the hindrance propagation backward could affect more trains and cause huge influence. Therefore, the negative influence of hindrance backward propagation was detailed and investigated in paper.

Just as the name implies, backward propagation means the influence of certain hindrance to the following-up trains. The attached following train will be hindered by this hindered train of initial hindrance if the buffer time between these two trains is not enough, comparing the length of initial hindrance. In this case, a new hindrance occurs to the next following train due to this initial hindrance. Furthermore, the hindered train with new propagating source may become a hindering train of other hindered trains. This situation may repeat itself between subsequent train and its attached trains so that the hindrance influence accumulates and continuously propagates backward. Figure 6 gives an example to describe the situation of hindrance backward propagation.

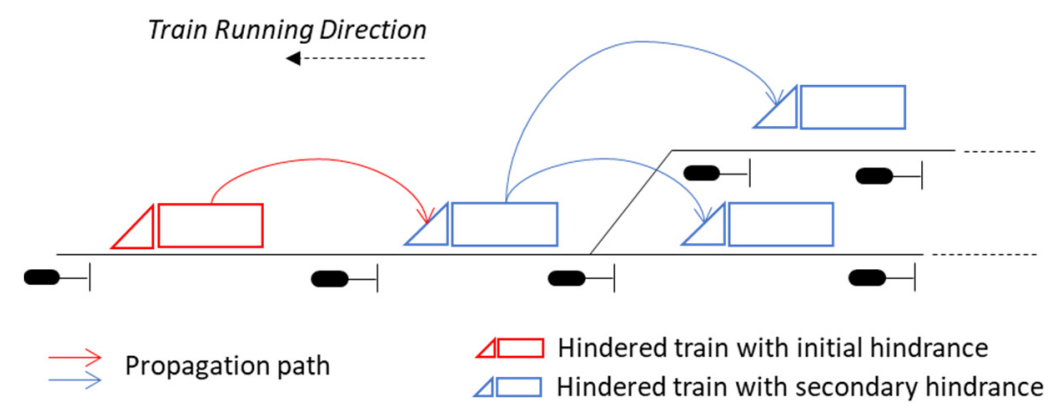

Figure 6. An example of backward hindrance propagation.

\subsection{Hindrance Propagation Process}

A tree structure would firstly be established for each initial hindrance to trace the hindrance propagation process between trains. As shown in Figure 7, the square nodes describe attributes of a hindrance, including the ID of hindered train, the component on which this hindrance happens, and the length of hindered time. Moreover, the link between two nodes describes the relationship between hindrances; in other words, it is the direct cause of hindrance, which can be described by individual hindrance. 


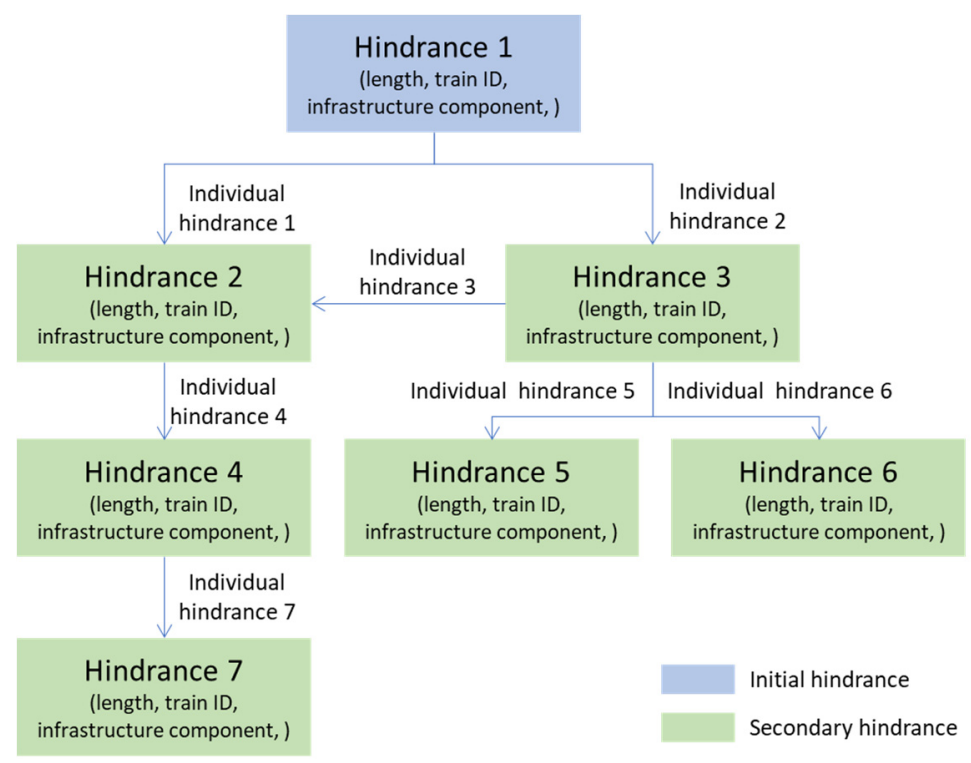

Figure 7. Tree structure of hindrance propagation.

As in the description of hindrance backward propagation, if the attached train is hindered by the hindered train of initial hindrance on the component for which the hindered component of initial hindrance acts as its potential hindering component, the new hindrance is the first negative influence of hindrance backward propagation.

Furthermore, the new hindrance can be treated as a new initial hindrance to be checked whether it is transformed to attached trains as first hindrance. Once there is a new secondary hindrance caused by the new initial hindrance, the new secondary hindrance is the influence of new initial hindrance in the same way. Forward pursuing, the new initial hindrance is caused by initial hindrance, so that the new secondary hindrance is also the secondary hindrance of initial hindrance, which should be calculated as the influence of initial hindrance.

Backward propagation is the repetition of these hindrance transformation processes. Moreover, the sequence of the transformations is called the hindrance propagation relationship of initial hindrance, in which the relationship is interrelated by hindered train and hindered component. The relationship ends up with the last secondary hindrance and is transformed to the attached trains.

Therefore, in the process of establishing the tree structure, the first step is to search all hindrances that occur in the whole network creating the nodes. Afterward, the link between these nodes would be made with the assistance of the individual hindrance by correlating the hindered train and the corresponding infrastructure component.

\subsection{Determine the Influence of Hindrance}

Based on this tree structure, the influence of hindrance that occurs on certain influence components can be further investigated and analyzed. When the conflict happens, the influence of hindrance caused by different choices could be predicted.

In this tree structure describing the prices hindrance propagation process with the extent and the depth, the extent is the total numbers of trains suffering secondary hindrances, and the depth is the total number of secondary hindrances corresponding to the longest path in hindrance propagation. Take Figure 7 as an example; in total, six trains are affected with the depth as 3 by the initial hindrance.

Besides, the overall influence of the hindrance backward propagation is the sum of all secondary hindrances, which can be calculated by the following equation:

$$
O H_{i, j}=\sum_{p=1}^{N} H_{p, q}
$$


where $\mathrm{OH}_{i, j}$ is the overall influence caused by hindered train $i$ on infrastructure component $j ; \mathrm{N}$ is the total number of trains affected by hindered train $i$ on infrastructure component $j$; and $H_{p, q}$ is the second hindrance caused by the hindered train $i$ on infrastructure component $j$.

It comes naturally to calculate the propagation rate, which is the ratio of overall influence. Then, the overall propagation rate is calculated as the ratio of overall influence caused by hindrance to its length. The computational formula is as follows:

$$
O P R_{i, j}=\frac{O H_{i, j}}{H_{i, j}}
$$

For a certain component, different lengths of hindrance appear on it during daily operation. Through the observation of huge data, the length of hindrance directly influences the extent and depth of the corresponding tree structure of hindrance propagation process. Even more critical is that these differences of propagation rates for various extents are so significant that they cannot be ignored for backward influence estimation. Therefore, a scatter diagram is drawn to interpret how overall influence changes with the length of initial hindrance on certain component in order to have a more specific understanding of the interrelationship between two variables. The function relationship between propagation rate and length of hindrance was investigated by regression algorithm. Finally, when hindrance happens on certain infrastructure, the influence of this hindrance could be predicted through this function to give dispatchers a support in dealing with conflict.

\section{Case Study}

\subsection{Research Area}

In this paper, the calculation and influence analysis of hindrance is carried out based on a reference network. The railway network consists of four railway stations, named AHX, EN, LBC, and BS, in which BS is a through station of two lines: one is between station AHX and station LBC, and the second line is between station LBC and station EN. The routes of trains between station LBC and EN and the routes of trains departing from AHX and leading to LBC, or in-verse, intersect at station BS.

According to train routes and request time of signals, the whole network is divided into 71 components which are the minimum exclusive infrastructure components of hindrance arisen, and the detail components' layout in the railway network of the institute example is shown in Figure 8. The calculation of hindrance is based on the components. In addition, the components between station LBC and station EN are double directions, and the others are one-direction components. After integration treatment, finally 62 components are studied in this example.

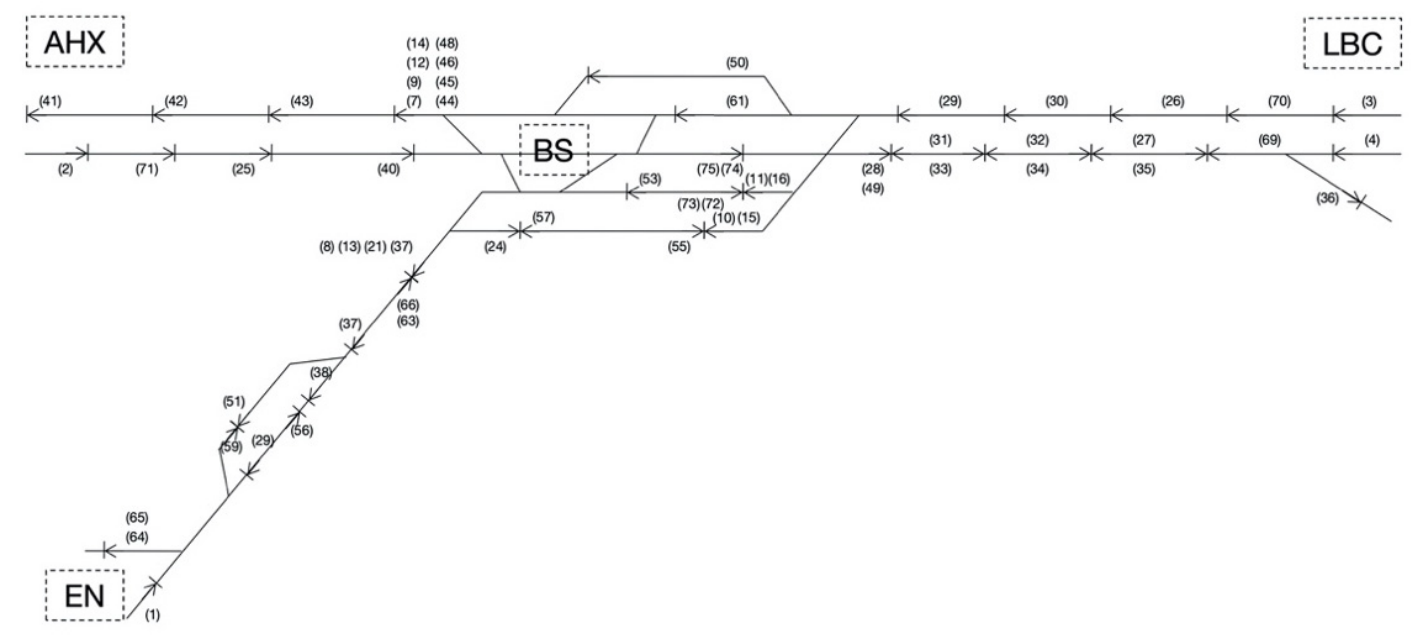

Figure 8. Layout of reference network with infrastructure component. 


\subsection{Data Processing}

The input for individual hindrance calculation is based on the micro simulation result of random generated timetables. The running conflicts in these random generated timetables are considered equivalent to the hindrances happening in conflict-free timetables. In this research, we used RailSys [22] to synchronous simulate the operation of trains in the reference railway network with the random timetables created by PULEIV [23] base on a basic operating program.

For this reference network, a total of 250 timetables are randomly generated. Each timetable has 144 trains operating for 6 hours. There trains are categorized into three types: FRz (long-distance passenger train), $\mathrm{NRz}$ (short-distance passenger train) and GV (freight train). The proportion of each type of train is $16.7 \%$ for FRz, 33.3\% for NRz, and 50\% for GV.

After each simulation, log files are generated in which the scheduled and real operational timetables are stored. Based on micro infrastructure modeling, the operating data of trains during the simulation is recorded for each component. The running time recorded is associated with the corresponding component.

Based on the simulation results, the individual hindrance occurs on each infrastructure component can be calculated precisely. It was found that the length of initial hindrance ranges widely on some infrastructure components. In addition, several seconds differences in length of initial hindrance will not result in great difference in backward propagation influence in real operation. Therefore, these initial hindrances are transformed into intervals with interval as $10 \mathrm{~s}$. For example, interval 6 represents the range between $50 \mathrm{~s}$ (including) and $60 \mathrm{~s}$ (not including). Consequently, the target of hindrance backward propagation modeling is to evaluate the influence of initial hindrance to the whole network through backward propagation responding to each hindrance interval.

\subsection{Results Analysis}

\subsubsection{The Hindrance Propagation Relationship}

The propagation relationship is a simple but powerful visual method to understand how an initial hindrance, in absence of other disturbance or schedule modification, creates backward propagation through the railway network. Figure 9 gives an example of hindrance propagation based on the simulation protocol.

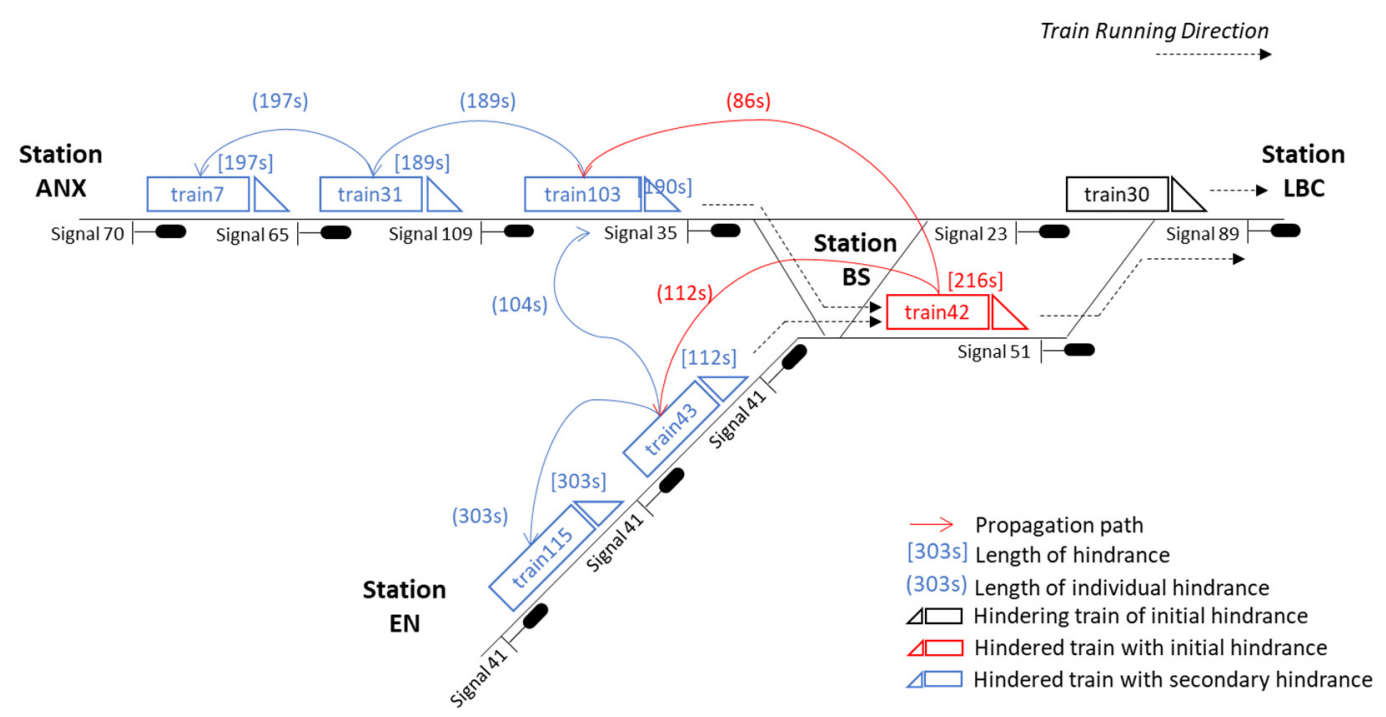

Figure 9. Hindrance backward propagation process of initial hindrance of train 42.

In this example, the initial hindrance occurs on component 73, and train 42 is hindered by train 30. Afterward, this hindrance propagates into two directions through train 103 and train 43. Finally, 
in total, five trains are affected by the initial hindrance of train 42 , and the hindrance propagation relationship is established in Figure 10.

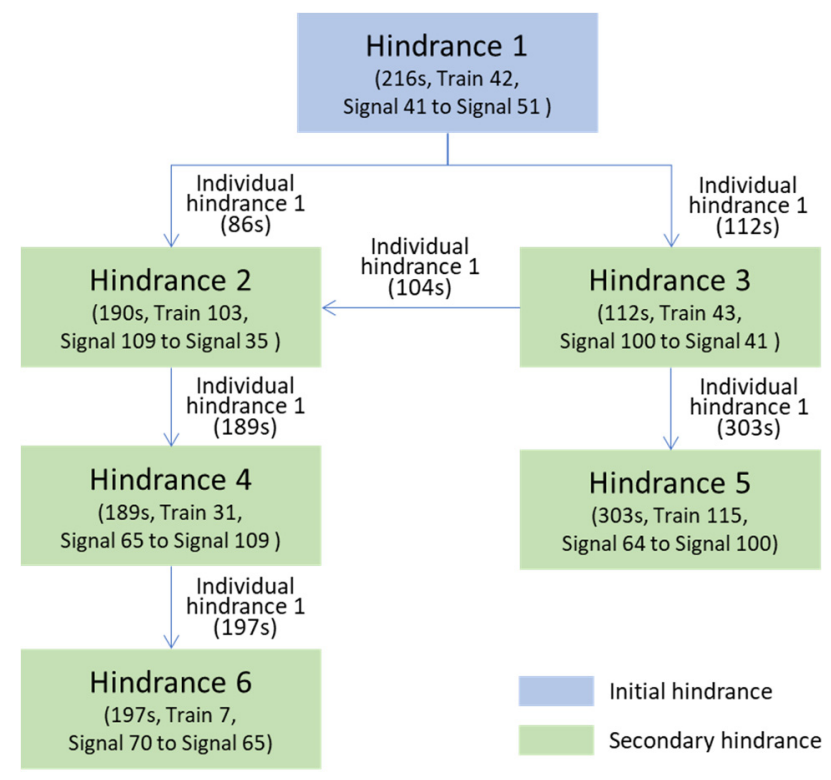

Figure 10. The propagation tree structure of initial hindrance of train 42 .

As shown in Figure 10, the initial hindrance occurs on component 73, in that train 42 is hindered by train 30 for $216 \mathrm{~s}$, which results in two paths' backward propagation with five secondary hindrances. For the left propagation path in Figure 10, train 103 is hindered by this initial hindrance for $190 \mathrm{~s}$ on component 40, which afterward causes secondary hindrances with the sequence: Train 31 is hindered for $189 \mathrm{~s}$, and train 7 is hindered for $197 \mathrm{~s}$. In this path, three secondary hindrances are produced with depth as 3 . For the other propagation path, the initial hindrance is transformed to train 43 , causing $112 \mathrm{~s}$ secondary hindrance on component 63 , which is continuously transformed to train 115 along with $303 \mathrm{~s}$ secondary hindrance. The depth of right path is 2 with two secondary hindrances. In summary, the influence of backward propagation for initial hindrance of train 42 occurred from signal 41 to signal 51 is totally $991 \mathrm{~s}$ with the extend as 5 and the depth as 3, corresponding to the left propagation path.

\subsubsection{The Influence of Hindrance Propagation}

Take initial hindrances on infrastructure component from signal 109 to signal 35 as an example to investigate the changing trend of overall influence on certain component responding to the length of hindrance.

For initial hindrances that occur on this component, a total of 910 hindrances are recorded. These hindrances range from $27 \mathrm{~s}$ to a maximum of $1225 \mathrm{~s}$. For each hindrance, the overall influence is computed through the tree structure, to trace the hindrance backward propagation. As mentioned in date processing, these records are spreading into sections with $10 \mathrm{~s}$ as interval (from interval 0 to interval 125) based on the length of hindrance. For each interval, the mean propagation rate calculated presents the overall influence in this interval.

The simulation results show that a maximum of two secondary hindrances were caused by these initial hindrances. Then, mean propagation rates were calculated for extent 0,1 , and 2 , and the corresponding mean propagation rates are $0,0.7852$, and 1.4873 . One more secondary hindrance is initiated; the influence of initial hindrance may have a big difference. Therefore, the mean value of propagation rate regarding extent is calculated in thesis. It proves that an initial hindrance can "snowball" through the entire railway network, and the secondary hindrances induced create significantly more impact than the initial hindrance itself. With the share of extent for hindrance intervals and the corresponding mean propagation rate in regard to hindrance interval, the overall 
propagation rate and overall influence for each interval on this component can be calculated according to Section 3.3. Figure 11 gives a scatter diagram, showing the relationship between overall influence and hindrance interval on component 40. It is clear that, with an increase in hindrance interval, there is a corresponding increase in overall influence. At the same time, the growth rate of overall influence increases with the length of hindrance.

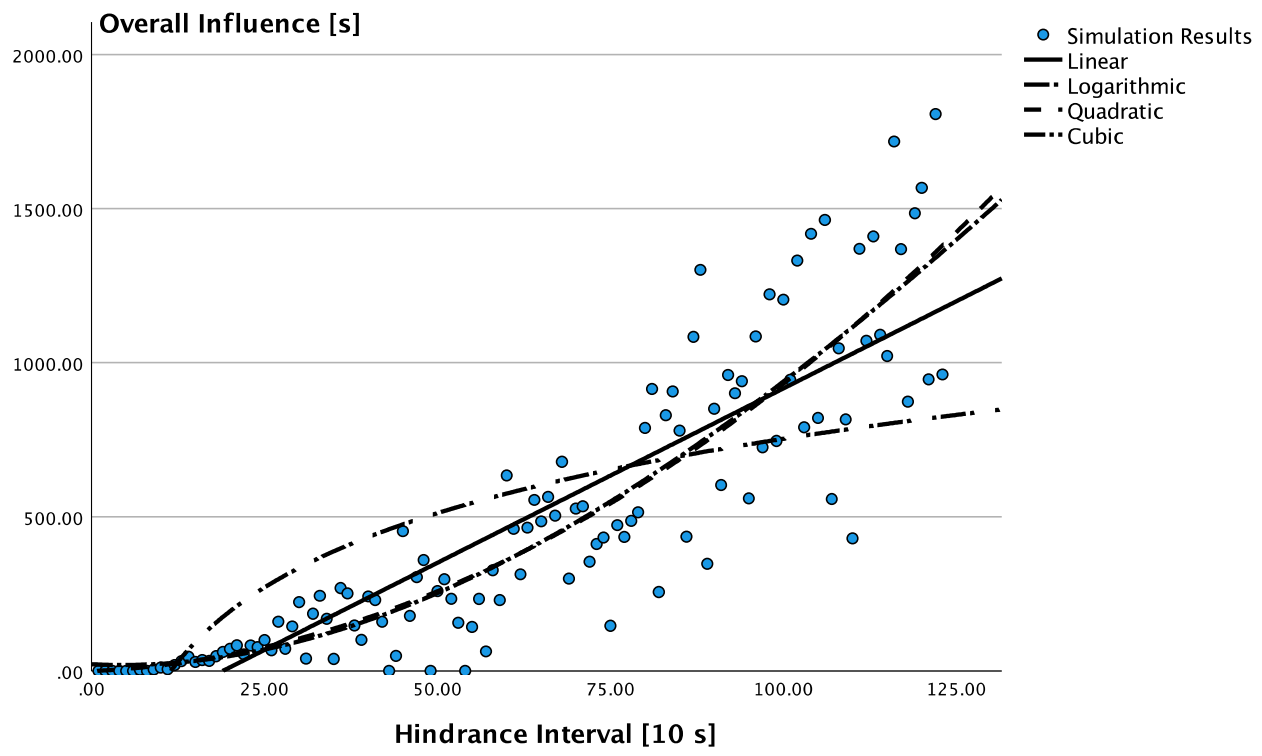

Figure 11. Scatter diagram for overall influence vs. the length of initial hindrance.

In order to further investigate the mathematical interrelationship between the length of hindrance and its overall influence of overall influence, a regression analysis was performed. Meanwhile, the overall influence prediction model from independent length of hindrance can be derived for a certain infrastructure component. Based on the increase trend of these scatters, linear, logarithmic, quadratic, and cubic models are chosen for the regression of the overall influence in the case study. We use maximum likelihood estimation to determine the related function parameters. Table 1 shows the results of the regression analysis for the infrastructure component 40, from signal 109 to signal 35. It can be found that the regression model quadratic model fit the simulation results best with $R$-Square as 0.805 . Compared with cubic model, the difference in $R$-Square is small. However, considering the computational complex, we think the quadratic model is better for describing the relationship between hindrance interval and overall influence of hindrance.

Table 1. Parameter estimates and model summary for regression analysis.

\begin{tabular}{|c|c|c|c|c|c|}
\hline \multirow{2}{*}{ Equation } & \multicolumn{3}{|c|}{ Parameter Estimates } & \multicolumn{2}{|c|}{ Model Summary } \\
\hline & Parameter & $B$ & $t$ & $R$-Square & $F$ \\
\hline \multirow{2}{*}{ Linear } & Constant & -234.73 & -5.259 & \multirow{2}{*}{0.764} & \multirow{2}{*}{377.52} \\
\hline & Var & 11.55 & 19.792 & & \\
\hline \multirow{2}{*}{ Logarithmic } & Constant & -1185.63 & -6.783 & \multirow{2}{*}{0.559} & \multirow{2}{*}{146.88} \\
\hline & $\operatorname{Ln}($ var $)$ & 429.92 & 10.981 & & \\
\hline \multirow{3}{*}{ Quadratic } & Constant & -0.36 & 0.0498 & \multirow{3}{*}{0.805} & \multirow{3}{*}{233.49} \\
\hline & Var & 1.03 & 5.043 & & \\
\hline & $\operatorname{Var}^{2}$ & 0.08 & -0.016 & & \\
\hline \multirow{4}{*}{ Cubic } & Constant & 21.61 & 0.282 & \multirow{4}{*}{0.806} & \multirow{4}{*}{164.352} \\
\hline & Var & -1.08 & -0.204 & & \\
\hline & $\operatorname{Var}^{2}$ & 0.13 & 1.261 & & \\
\hline & $\operatorname{Var}^{3}$ & 0.00 & -0.436 & & \\
\hline
\end{tabular}


It is found that the propagation rate increases along with the increase of extent, as demonstrated above. However, the value of propagation rate, as well as the magnitude of the increase, differs from hindered component to hindered component. Therefore, regression analyses were also done for other infrastructure components based on the quadratic model, and the results are shown in Table 2 . Moreover, it can be found that the overall influence presents a gradual increase with the increase of length of hindrance. The fit goodness of the quadratic model is always relative higher among these models.

Table 2. Examples of regression analysis results for other components.

\begin{tabular}{|c|c|c|c|c|c|c|}
\hline \multirow{2}{*}{$\begin{array}{l}\text { Infrastructure } \\
\text { Component }\end{array}$} & \multirow{2}{*}{$\begin{array}{c}\text { Count of } \\
\text { Hindrance (s) }\end{array}$} & \multirow{2}{*}{$\begin{array}{l}\text { Length of } \\
\text { Hindrance (s) }\end{array}$} & \multicolumn{3}{|c|}{ Parameter Estimates } & \multirow{2}{*}{$R$-Square } \\
\hline & & & Constant & b1 & b2 & \\
\hline $\begin{array}{c}26 \text { (signal } 70 \text { to } \\
\text { signal } 65)\end{array}$ & 803 & 47 to 12,253 & 116.64 & 16.24 & 0.002 & 0.86 \\
\hline $\begin{array}{c}25 \text { (signal } 65 \text { to } \\
\text { signal 109) }\end{array}$ & 572 & 49 to 1225 & -48.21 & 3.36 & 0.034 & 0.76 \\
\hline $\begin{array}{l}40 \text { (signal } 109 \text { to } \\
\text { signal 35) }\end{array}$ & 910 & 27 to 1225 & -0.36 & 1.03 & 0.083 & 0.80 \\
\hline $\begin{array}{c}30 \text { (signal } 84 \text { to } \\
\text { signal } 83)\end{array}$ & 828 & 49 to 12,325 & 289.19 & 15.41 & 0.010 & 0.72 \\
\hline $\begin{array}{c}29 \text { (signal } 83 \text { to } \\
\text { signal 10) }\end{array}$ & 804 & 50 to 12,308 & 882.68 & 12.17 & 0.020 & 0.69 \\
\hline $\begin{array}{l}57 \text { (signal } 10 \text { to } \\
\text { signal } 48 \text { ) }\end{array}$ & 97 & 48 to 12,526 & 2473.54 & -7.57 & 0.192 & 0.91 \\
\hline $\begin{array}{l}74 \text { (signal } 35 \text { to } \\
\text { signal 23) }\end{array}$ & 486 & 20 to 415 & -93.75 & 17.75 & -0.028 & 0.77 \\
\hline $\begin{array}{l}75 \text { (signal } 41 \text { to } \\
\text { signal 23) }\end{array}$ & 117 & 53 to 301 & 215.99 & -38.59 & 2.156 & 0.77 \\
\hline
\end{tabular}

Combining with the layout of reference network, we see the relatively small influence exists on components related to route between AHX and LBC. The probability of hindrance happening on infrastructure component 57 is relatively low, but it has the maximum propagation rate. This is because of the intersection of routes from LBC to EN and routes leading to LBC. The estimated parameters vary among these infrastructure components due to their position and characteristic in the infrastructure. Moreover, the different locations of these infrastructure components induce the various in the amount and structure of different train types through the infrastructure component. These factors induce different utilization efficiencies and occupation times. Normally, the infrastructure component, which has more conflict routings, releases a greater impact on the whole network.

We have to point out that these functions are applicable only for our case with specific conditions. The layout of the whole network, the characteristics of certain infrastructure component, the traffic flow, the train mixture, and other parameters will affect the final regression function. However, having simulated or empirical data, this proposed method can be used for any case, to investigate and predict the overall influence of hindrance on each infrastructure component.

Up to now, the influence of hindrance is done for the same traffic flow run 24 trains per hour with the same train structure. In order to analyze the influence of train density to the relationship between overall influence through hindrance backward propagation in the reference railway network, the hindrance propagation model with traffic flow 36 train/hour, 48 train/hour, and 60 train/hour are compared for infrastructure component 40 from signal 109 to signal 35.

In Table 3, it has been found that the mean propagation rate is increased along with train density for extents 1 and 2, in which case the initial has a negative impact on the whole railway network. Moreover, the margin of increase between 48 train/hour and 60 train/hour is bigger than that between 36 train/hour and 48 train/hour. Figure 12 shows the scatter diagram describing the relationship between overall influences of hindrance backward propagation on component 40 . Comparing the regression result of hindrance propagation modeling, it is found that the curve with 60 train/hour is 
steeper than that of 36 train/hour and 48 train/hour, which proves that the impact of initial hindrance becomes greater with increasing density once again. That is because of the insufficient buffer time between sequential trains due to the high train density.

Table 3. Comparison of mean propagation rate for different train density (component 40).

\begin{tabular}{cccc}
\hline \multirow{2}{*}{ Traffic Flow (train/hour) } & \multicolumn{4}{c}{ Mean Propagation Rate with Regard to Extent } \\
& $\mathbf{0}$ & $\mathbf{1}$ & $\mathbf{2}$ \\
\hline 36 & 0 & 0.72 & 1.33 \\
48 & 0 & 0.79 & 1.49 \\
60 & 0 & 1.07 & 2.02 \\
\hline
\end{tabular}

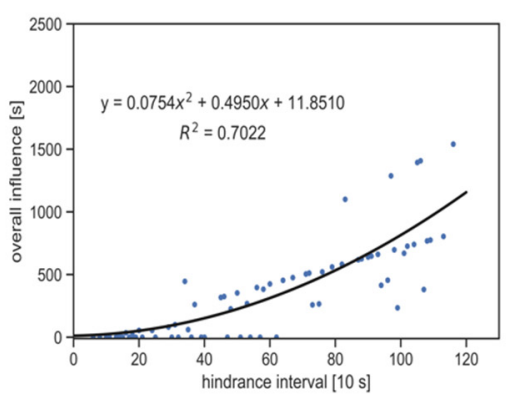

(a)

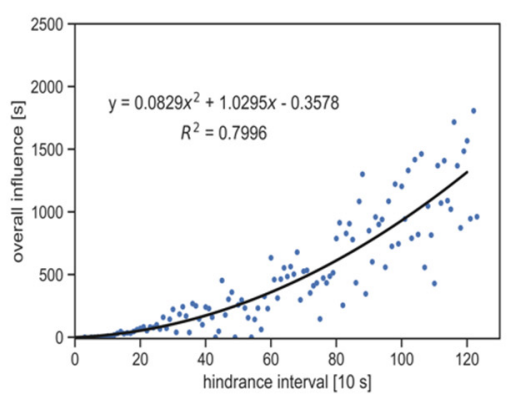

(b)

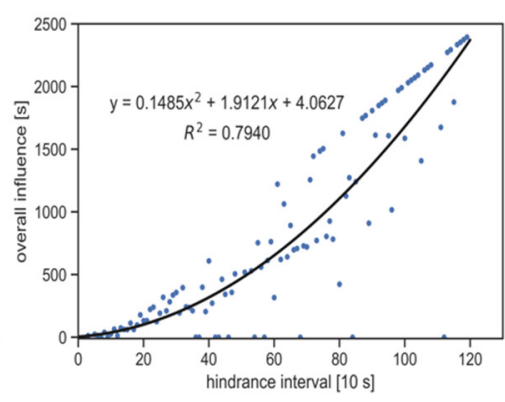

(c)

Figure 12. Comparison of overall influence of hindrance propagation with different traffic flow (component 40): (a) 36 train per hour, (b) 48 train per hour, and (c) 60 train per hour.

\subsection{Discussion}

The tree structure relationship could describe the propagation paths of a hindrance based on individual hindrance. The propagation rate and overall influence for each hindrance is calculated to present the influence of initial hindrance to the whole network. In the case study of reference network, it is proved that an initial hindrance can propagate to other trains, creating an even more significantly negative impact on the entire network than the initial hindrance itself. Its effect increases as its length increased. Using the regression analysis, the interrelationship between hindrance and overall influence is identified, and the overall influence prediction models from length of hindrance are derived. The results show that quadratic model fits hindrance backward propagation on most components well. Meanwhile, this relationship is also affected by the location of the infrastructure component, as well as the amount of traffic flow in the network. The hindrance happens on the infrastructure component having more conflict routings always releases a greater negative influence. At the same time, with high density of traffic flow, the hindrance will induce larger overall influence to the network.

\section{Conclusions and Future Research}

Hindrance is an important issue that should be considered in railway dispatching. A small individual hindrance will aggregate and propagate, creating a great influence to the entire network, so the hindrance cannot be ignored. This paper proposed a new perspective to analyze the influence of operation disturbances to the whole network, based on hindrance. Firstly, a new method was developed to calculate the length of hindrance, and the relationship between hindrances was clarified by individual hindrances. Secondly, tree structure was proposed to analyze the hindrance propagation process in the entire network. A regression analysis was done individually for certain infrastructure components, to figure out the relationship between the overall influence of hindrance and the length of hindrance. When a hindrance occurs, or a dispatching decision has to be made between two trains, the influence of hindrance could be estimated with these propagation models. 
In the experiment of reference network, a hindrance propagation model was made for each infrastructure component. The results demonstrated that the hindrance will indeed propagate and will later create a huge negative influence in the whole network. The impact is positively related to the length of the initial hindrance. In addition, the denser the railway network is, the greater the hindrance propagation rate is on components. The example proved a new method can be utilized to help dispatch find the influence of each hindrance.

However, a lot of work still can be done in future. The relationship between hindrance influence and the length of initial hindrance, train density of railway system, and the location of hindrance occurrence is studied. However, as we know, the generation and propagation of hindrance is still influenced by other factors, like network typology, train mixture, driver behavior, and so on. The research of multiple impact factors could be done in the future. The application of these methods, as well as their effectiveness and accuracy, still needs further testing.

Author Contributions: Conceptualization, T.T. and N.C.; methodology, T.T. and N.C.; formal analysis, N.C.; resources, C.G.; writing-original draft preparation, N.C.; supervision, T.T. and C.G. All authors have read and agreed to the published version of the manuscript.

Funding: This research was funded by Beijing Postdoctoral Research Foundation.

Conflicts of Interest: We declare that we have no financial or personal relationships with other people or organizations that could inappropriately influence our work. There is no professional or other personal interest of any nature or kind in any product, service, and/or company that could be construed as influencing the position presented in, or the review of, the manuscript entitled.

\section{References}

1. Olsson, N.O.E.; Haugland, H. Influencing factors on train punctuality Results from some Norwegian studies. Trans. Policy 2004, 11, 387-397. [CrossRef]

2. Palmqvist, C.W.; Olsson, N.O.E.; Hiselius, L.W. The Planners' Perspective on Train Timetable Errors in Sweden. J. Adv. Transp. 2018, 2018, 1-17. [CrossRef]

3. Pachl, J. Railway Operation and Control, 3rd ed.; VTD Rail Pub: Mountlake Terrace, WA, USA, 2015.

4. Krmac, E.; Djordjević, B. An evaluation of train control information systems for sustainable railway using the analytic hierarchy process (AHP) model. Eur. Transp. Res. Rev. 2017, 9, 1-17. [CrossRef]

5. Schittenhelm, B.H. Quantitative Methods for Assessment of Railway Timetables. Ph.D. Thesis, Technical University of Denmark, Lyngby, Denmark, 2013.

6. Goverde, R.M.P.; Odijk, M.A. Performance evaluation of network timetables using PETER. In Computers in Railways; Allan, J., VIII, Andersson, E., Eds.; WIT Press: Southampton, UK, 2002; Volume 61, pp. 1-10.

7. Liu, F.; Xu, R.; Fan, W.; Jiang, Z. A Data Analytics Approach for Train Timetable Performance Measures Using Automatic Train Supervision Data. IET Intell. Transp. Syst. 2018, 12, 1-10. [CrossRef]

8. Peng, Q.; Bao, J.; Wen, C.; Feng, L. Evaluation theory and method of high-speed train diagrams. J. Southwest Jiaotong Univ. 2013, 48, 969-974.

9. Harrod, S.; Cerreto, F.; Nielsen, O.A. A closed form railway line delay propagation model. Transp. Res. Part C Emerg. Technol. 2019, 102, 189-209. [CrossRef]

10. Jin, B.; Feng, X.; Wang, Q.; Wang, X.; Liu, C. Improving Timetable Robustness through Optimal Distribution of Runtime Supplement; ITSC 2019: Auckland, New Zealand, 2019.

11. Vansteenwegen, P.; van Oudheusden, D. Developing railway timetables which guarantee a better service. Eur. J. Oper. Res. 2006, 173, 337-350. [CrossRef]

12. Goverde, R.M.P. Railway timetable stability analysis using max-plus system theory. Transp. Res. Part B Methodol. 2017, 41, 179-201. [CrossRef]

13. Goverde, R.M.P. Synchronization Control of Scheduled Train Services to Minimize Passenger Waiting Times. Ph.D. Thesis, Delft University of Technology Delft, Delft, The Netherlands, 1998.

14. Li, X.; Martin, U.; Oetting, A.; Nachtigall, K. Methodik zur effizienten marktgeeigneten Trassenbelegung im spurgeführten Verkehr. ETR 2017, 6, 56-64.

15. Goverde, R.M.P. A delay propagation algorithm for large-scale railway traffic networks. Transp. Res. Part C Emerg. Technol. 2010, 18, 269-287. [CrossRef] 
16. Martin, U. Verfahren zur Bewertung von Zug- und Rangierfahrten bei der Disposition. Ph.D. Thesis, TU Braunschweig, Braunschweig, Germany, 1995.

17. Meester, L.E.; Muns, S. Stochastic delay propagation in railway networks and phase-type distributions. Transp. Res. Part B Methodol. 2007, 41, 218-230. [CrossRef]

18. Büker, T.; Seybold, B. Stochastic modelling of delay propagation in large networks. Int. J. Rail Transp. 2012, 2, 34-50. [CrossRef]

19. Yuan, J. Stochastic Modelling of Train Delays and Delay Propagation in Stations. Ph.D. Thesis, Delft University of Technology Delft, Delft, The Netherlands, 2006.

20. Corman, F. Real-time Railway Traffic Management: Dispatching in Complex, Large and Busy Railway Networks. Ph.D. Thesis, The Netherlands Trail Research School, Delft, The Netherlands, 2010.

21. Warninghoff, C.R.; Bendfeldt, J.P. Infrastrukturbezogenen Auswertung von Betriebssimulationen in der Eisenbahnbetriebswissenschaft. ETR 2004, 6, 363-370.

22. RailSys 7.0; RMCon (Rail Management Consultants): Hannover, Germany, 2010; Available online: https: //www.rmcon-int.de/railsys-en/ (accessed on 5 July 2020).

23. PULEIV 3.0; IEVVWI (Institute of Railway and Transport): Stuttgart, Germany, 2013; Available online: https://www.vwi-stuttgart.de/Software-Puleiv.html (accessed on 5 July 2020).

(C) 2020 by the authors. Licensee MDPI, Basel, Switzerland. This article is an open access article distributed under the terms and conditions of the Creative Commons Attribution (CC BY) license (http://creativecommons.org/licenses/by/4.0/). 